\section{Migraine with aura and persistent foramen ovale}

PT Wilmshurst

\begin{abstract}
The association between migraine with aura and persistent foramen ovale (PFO), as well as other right-to-left shunts, is described. A hypothesis that might explain this association is discussed. Observational studies suggested that when a PFO is closed patients who have migraine with aura are usually improved. The Migraine Intervention with STARFlex Technology (MIST) Trial was a randomised double-blind trial in patients with severe intractable migraine with aura and a moderate-large PFO that compared implantation of STARFlex devices with the intention of closing their PFO versus a sham procedure. It was hoped that the trial would demonstrate whether PFO closure would cure migraine. A series of problems in design and execution of the trial are discussed.

Eye (2018) 32, 184-188; doi:10.1038/eye.2017.269; published online 8 December 2017
\end{abstract}

In order to diagnose migraine and migraine with aura a patient must satisfy specific headache criteria and neurological criteria for aura. Diagnosis does not mean that migraine is a disease. It is a complex symptom.

Angina pectoris is also a complex symptom with specific criteria (specifically chest or throat discomfort triggered by exertion) but many diseases cause the symptom. It is probable that migraine and migraine with aura are symptoms of more than one disease.

Approximately $13 \%$ of the population have migraine and about one-third of them, $4 \%$ of the population, have migraine with aura. Migraine is more common in women than men.

People with migraine with aura have an increased prevalence of PFO than controls, but people with migraine without aura do not have an increased prevalence of PFO. ${ }^{1,2}$

About $27 \%$ of the adult population have a $\mathrm{PFO}$, but in most cases the defect has a small diameter. ${ }^{3}$ A PFO, particularly when large, will allow blood in the right atrium to cross the atrial septum to the left atrium to bypass the pulmonary capillary filter. The magnitude of a right-to-left shunt across the PFO is related to its diameter, as well as other factors such as the pressure gradient between the atria. The size of the shunt across a PFO assessed using bubble contrast echocardiography is related to the prevalence of migraine with aura. ${ }^{4}$ Migraine with aura occurs in $4 \%$ in people with a small shunt-equal to the prevalence in the general population. Twenty-five percent in people with a medium size shunt and $53 \%$ in those with a large shunt have migraine with aura. ${ }^{4}$

Even when a shunt across a PFO is large it rarely cause desaturation of arterial haemoglobin, but shunts large enough to cause arterial desaturation are also associated with migraine with aura. Fifty-nine percent of patients with cyanotic congenital heart disease have migraine with aura (distinct from the hyperviscosity headaches these patients get as a result of polycythaemia). ${ }^{5}$ Fifty percent of patients with hereditary haemorrhagic telangiectasia, which is associated with a high prevalence of pulmonary arteriovenous malformations, have migraine with aura. ${ }^{6}$

Overall, the data suggest that prevalence of migraine with aura increases with the size of right-to-left shunts, whatever their cause. This resulted in the hypothesis that substances that circumvent the pulmonary filter by crossing the shunt may be responsible for precipitating attacks of migraine with aura. ${ }^{7}$

In total, $40-50 \%$ of people with the largest shunts do not get migraine with aura, which might be because they do not produce the trigger substance in venous blood or their brains are less susceptible to the trigger. In addition, about half the people with migraine with aura have no shunt. They presumable have a different mechanism, unrelated to right-to-left shunting, as the cause. It follows that some people that have a right-to-left shunt will have migraine with aura as a result of the other mechanism (unrelated to a right-to-left shunt). The data 
suggest that such coincidence is most likely when the shunt size is small.

People with migraine with aura have high incidences of shunt related events. They have an incidence of stroke that is 2.88 times that of people without migraine. Women with migraine with aura on the combined oral contraceptive pill have a risk of stroke that is increased 8.72-fold, which may be the result of combining the increased prevalence of large shunts (indicated by their migraine with aura) with increased risk of venous thrombosis from the contraceptive pill to cause paradoxical thromboembolism. ${ }^{8}$

The majority of episodes of neurological, cardiovascular and cutaneous decompression illness are the result of paradoxical gas embolism-venous bubbles that form during decompression circumventing the lung filter by crossing a PFO or other right-to-left shunt. ${ }^{9,10}$ People with migraine with aura have a greatly increased risk of decompression illness. ${ }^{11}$ This increased risk appears to be confined to migraineurs with the largest shunts. ${ }^{4}$ As a result, the medical standards for divers in many countries advise that a history of migraine with aura is an indication to check prospective divers for a PFO so that they can be advised about their increased risks when diving. ${ }^{12}$

It is important to appreciate that the size of the shunt across a PFO is related to diameter of the defect. Although $27 \%$ of the population have a PFO, the shunt across most is small and only $1.3 \%$ of the population have a PFO $1 \mathrm{~cm}$ diameter or larger. ${ }^{3}$ In contrast, nearly all divers who suffer shunt-mediated decompression illness have a large shunt and the majority have a PFO that is $1 \mathrm{~cm}$ diameter or greater. ${ }^{13}$

Migraine often appears to run in families. In many families, the presence of a large PFO is dominantly inherited and co-segregates with affected individuals having migraine with aura. ${ }^{14,15}$

Prevalence and size of PFOs are equal in men and women, but migraine is three times more frequent in women than men. It is not known whether the migraine excess in women is in those with a large PFO or without a PFO. In some women, migraine attacks appear to be influenced by hormone changes: often starting or increasing at puberty and being influenced by the menstrual cycle, pregnancy and the menopause.

Pulmonary shunts are more frequent in women and their size can change with the levels of female hormones. It is not known whether the variability of pulmonary shunts influences migraine incidence in women, or whether different levels of trigger substances or tissue susceptibility to the triggers have a role in the observed excess incidence in women.

Many observational studies have shown that when large PFOs are closed in patients who have had stroke or decompression illness that is believed to be the result of paradoxical embolism (thrombus or gas), the patients have a high prevalence of migraine before the procedure and $\sim 80 \%$ report that their migraine symptoms were improved after the closure procedure. ${ }^{16-21}$ Migraine with aura is also improved by occlusion of pulmonary arteriovenous malformations. ${ }^{22}$

If a trigger substance is bypassing the lungs, what is it? One possibility is 5-hydroxytryptamine (5HT or serotonin). $5 \mathrm{HT}$ is present in the gastrointestinal tract (where it is required for motility), in platelets (which release it when activated to cause vasoconstriction and platelet aggregation), and in the brain (where it is a neurotransmitter). There is evidence that $5 \mathrm{HT}$ has a role in migraine and drugs that act on $5 \mathrm{HT}$ receptors are used to treat migraine. ${ }^{23}$ Virtually all the 5HT in venous blood is removed by passage through the lungs, probably by monoamine oxidase. ${ }^{24}$

If $5 \mathrm{HT}$ is the trigger substance in some migraineurs, high venous concentrations may arise as a result of platelet activation. In a randomised study of UK doctors prophylactic dose aspirin, which has an antiplatelet action, reduced migraine compared with controls. ${ }^{25}$

Interventions that activate platelet downstream from the lungs, such as implanting a prosthetic aortic or mitral valve, can trigger migraine with aura for the first time in people who have no shunt. Closure of a PFO or an atrial septal defect can also precipitate or exacerbate migraine with aura. ${ }^{16}$ These procedures involve implanting a double disc device across the defect in the atrial septum, with one disc in each atrium. So, at the end of the procedure there is a disc in the left atrium, which can active platelets downstream from the lungs. The increased propensity to migraine with aura after PFO closure lasts a few weeks and ceases about the time when the occlusion device is covered with endothelial growth and is no longer a site for platelet activation. Migraine with aura soon after PFO closure is virtually abolished if the patient is given clopidogrel $75 \mathrm{mg}$ daily for the first 4 weeks after the closure procedure. ${ }^{26}$

These observations resulted in the MIST Trial, which it was hoped would determine whether PFO closure would cure migraine with aura. ${ }^{27}$ It was a multicentre, randomised, double-blind, controlled trial. NMT Medical (Boston, MA, USA) sponsored the trial. The West Midlands Multicentre Research Ethics Committee approved it.

Patients with severe migraine with aura that was refractory to medical treatment were first assessed by a neurologist/headache specialist. Those that were eligible were referred to one of two centres for an initial contrast echocardiogram to determine whether they had a moderate or large shunt across a PFO. Those that did were eligible for the next stage of the trial and were 
referred to one of six interventional cardiology centres where they were randomised when under general anaesthetic to either implantation of a STARFlex device, with the intention of closing their PFO or to a sham procedure.

The aim was to randomise 75 patients to each arm of the trial. Neither the patients nor the neurologists / headache specialists that performed patient follow-up at monthly intervals for 6 months were told whether patients had had a STARFlex implant or a sham procedure. The first 3 months after the procedure were the healing period (when migraine might be expected to increase). The second 3 months were the analysis period and were used to compare the two arms of the trial using an intention to treat analysis.

I was the trial's principal cardiologist. I strongly advised NMT before the protocol was finalised that only patients with large shunts across a PFO should be included, because prevalence of migraine with aura is related to shunt size. Inclusion of patients with smaller shunts increased the chance that migraine with aura and a PFO were coincidental rather than causal. I had also strongly advised that the trial needed a single echocardiography core laboratory. My advice was rejected.

The results of the initial contrast echocardiograms at the two hospitals are shown in Table 1. The overall percentage of patients that had a moderate or large PFO was more than five times greater than in the general population and the percentage of patients with large pulmonary shunts was considerably greater than in the general population (it is usually much $<1 \%$ ). Three patients found to have atrial septal defects had those closed outside the trial.

There were important differences between the findings at the two hospitals that have relevance to the outcome of the trial. At the Royal Shrewsbury Hospital (RSH) there were more small shunts and many more pulmonary shunts, but fewer moderate-large PFO than at the Royal Brompton Hospital (RBH).

Of the 163 patients eligible for randomisation to implantation of a STARFlex device, 16 withdrew. Hence
74 patients were randomised to STARFlex implantation and 73 to a sham procedure. RSH and RBH each referred patients for randomisation procedures at three interventional cardiac centres (six centres in total).

At the end of the trial the patients with an implant had a repeat contrast echocardiogram. Against the advice of the steering committee, NMT insisted that the implanting cardiologists should perform the final contrast echocardiograms. The steering committee objected that the implanting cardiologists were interventionists and not experts on echocardiography and that they had operator bias and would be assessing their own handiwork. Also, that performance of the initial and final echocardiograms at the same sites would insure that the performance techniques were identical and so make comparisons in individual patients before and after STARFlex implantation reliable. However NMT agreed to the steering committee's advice that I, as the cardiologist with the most experience of contrast echocardiography and not involved in STARFlex implantations, should review the recordings of the final contrast echocardiograms.

The primary outcome, freedom from attacks of migraine during the analysis period, was identical in the two arms. Only three patients in each arm were migraine free. However, people viewing NMT's website might have believed that the trial had shown improvement from STARFlex implantation. Patients in the trial were identified outside the treating hospitals only by an alphanumeric code. Somehow, NMT was able to contact the three patients that were migraine free after a STARFlex implantation and their photographs and names appeared on NMT's website and in NMT's annual report together with their testimonials saying that they were migraine free after the implantation. (There were no statements from the three migraine-free patients who had sham procedures or the patients whose migraine was not improved or was worse after the implantation). Even more surprising was that one of the three patients who were migraine free after the STARFlex implant was pictured on the NHS website of the Royal Brompton Hospital with her testimonial to the treatment and the statement 'This is significant news for migraine sufferers.

Table 1 Number of patients (\%) with right-to-left shunts at the two hospital that screened patients with migraine with aura for shunts in the MIST Trial

\begin{tabular}{|c|c|c|c|}
\hline Size and type of shunt & Royal Brompton Hospital & Royal Shrewsbury Hospital & Both hospitals combined \\
\hline No shunt & $85(39.7 \%)$ & $87(39.9 \%)$ & $172(39.8 \%)$ \\
\hline Small shunt & $33(15.4 \%)$ & $39(17.9 \%)$ & $72(16.7 \%)$ \\
\hline Large pulmonary & $6(2.8 \%)$ & $16(7.3 \%)$ & $22(5.1 \%)$ \\
\hline Atrial septal defect & $3(1.4 \%)$ & 0 & $3(0.7 \%)$ \\
\hline Moderate-large PFO & $87(40.7 \%)$ & $76(34.9 \%)$ & $163(37.7 \%)$ \\
\hline Total & $214(100 \%)$ & $218(100 \%)$ & $432(100 \%)$ \\
\hline
\end{tabular}


For the first time this study has shown that closing a PFO can have a substantial effect in reducing the symptoms for patients with severe migraine.' The hospital management removed the webpage after I complained that the information was wrong.

A paper reporting results of the MIST Trial was published in Circulation, but it was so inaccurate that another member of the steering committee, Dr Simon Nightingale, and I refused to be authors of the paper. ${ }^{27}$ That resulted in NMT instructing their lawyers to sue both Dr Nightingale and me for libel. They did not serve the claim on Dr Nightingale, but sued me four times. The claims lasted $\sim 4$ years, until NMT went into liquidation in $2011 .^{28}$

During those 4 years, I convinced the editors of Circulation of errors in the paper and they publish a 700 words correction, a data supplement and a new version of the paper. ${ }^{27}$ The first author of the paper and principal headache specialist in the MIST Trial was Dr Andrew Dowson. I reported him to the General Medical Council alleging misconduct in the MIST Trial. He was found guilty of misconduct including two counts of dishonesty by a Fitness to Practise Panel in $2015 .{ }^{29}$ His appeal was rejected by the High Court and he was suspended from the Medical Register. ${ }^{29}$ At the hearings, it was shown that when the initial contrast echocardiograms showed a very high prevalence of large shunts, Dr Dowson had bought shares in NMT during the trial. NMT also paid him large sums of money. He disclosed no conflicts of interest in the paper. $^{27}$

The legal fog that engulfed the MIST Trial means that we may never understand why implantation of a STARFlex device in patients with severe migraine did not replicate the observed improvement in migraine seen in patients who had PFO closure following paradoxical embolism. It may be that in the latter group there was a large placebo effect. The difference may be related to the greater severity of migraine in the MIST Trial patients. Another possibility is that patient selection and trial design had deficiencies.

In the MIST Trial, 74 patients were randomised to STARFlex implantation. One patient was withdrawn at the time of the implantation procedure but before implantation because of a cardiac complication. That patient had her contrast echocardiogram at RSH. Five other patients, all from $\mathrm{RBH}$, were withdrawn at the time of the procedure because a PFO could not be found. Usually crossing a PFO in patients having the procedure is simple because the defect is large $(\sim 1 \mathrm{~cm}$ diameter). When I reviewed their contrast echocardiograms from $\mathrm{RBH}$ it was clear that pulmonary shunts had been misdiagnosed as shunts across a PFO. Three other patients who had had STARFlex implantation also withdrew from the trial at a later stage. The analysis by intention to treat meant that these nine patients were judged to have failed to improve. Indeed, it is difficult to see how those with pulmonary shunts could improve if those shunts were implicated in their migraine with aura and were unchanged by the procedure.

The confounding caused by pulmonary shunts did not end there. In another patient who had a contrast echocardiogram at $\mathrm{RBH}$ and in whom a PFO could not be crossed, the interventional cardiologist performed a transseptal puncture (ie, perforated the atrial septum) in order to implant a STARFlex device. That was not disclosed in the paper in Circulation. In another from the $\mathrm{RBH}$, the atrial septum was crossed with great difficulty using a fine coronary angioplasty wire and the size of the hole was too small to measure. A further six patients (five from $\mathrm{RBH}$ ) had a PFO between 2 and $4 \mathrm{~mm}$ diameter. Defects of those sizes are very unlikely to have clinical significance. It therefore appears that 17 of 74 (23\%) patients randomised to PFO closure either had a pulmonary shunt instead of a PFO or a small PFO with either an insignificant shunt or a combination of shunting across both a PFO and a pulmonary shunt.

At the end of the analysis period, 65 patients (ie, the 74 randomised to STARFlex implantation minus the nine withdrawn) had a contrast echocardiogram by the implanting cardiologists. They reported that four had a significant residual shunt.

Recordings of 64 of the contrast echocardiograms were sent to me for review. (One contrast echocardiogram could not be found.) I reported that 28 records were of such poor quality that it is impossible to exclude a significant residual shunt. In 15, I could see no evidence of a significant residual shunt. Twenty-one recordings showed a significant residual shunt and I reported that 13 were atrial (ie, residual shunts across a PFO), 5 were pulmonary, and in 3 cases I was uncertain whether the shunts were atrial or pulmonary.

Overall it appears that many patients in the MIST Trial had shunts that were predominantly pulmonary rather than across a PFO. It also appeared that when there was shunting across a large PFO on the initial contrast echocardiogram, the STARFlex device often failed to seal it. The STARFlex device successfully closed small PFOs, which may have been coincidental to another cause of migraine, but failed to close large PFOs. As a result, the role of shunting in migraine with aura remains uncertain.

\section{Conflict of interest}

The author declares no financial conflicts of interest. His involvement in the MIST Trial is detailed in this article, but it included him being the principal cardiologist in the trial, being sued for libel by the sponsor of the trial (until the litigation ended when the corporation went into 
liquidation), and reporting the trial's principal headache specialist to the General Medical Council, where the principal headache specialist was found guilty of dishonesty in the trial and was suspended from the Medical Register.

\section{References}

1 Del Sette M, Angeli S, Leandri M, Ferriero G, Bruzzone GL, Finocchi $\mathrm{C}$ et al. Migraine with aura and right-to-left shunt on transcranial doppler: a case-control study. Cerebrovasc Dis 1998; 8: 327-330.

2 Anzola GP, Magoni M, Guindani M, Rozzini L, Dalla Volta G. Potential source of cerebral embolism in migraine with aura. A transcranial doppler study. Neurology 1999; 52: 1622-1625.

3 Hagen PT, Scholz DG, Edwards WD. Incidence and size of patent foramen ovale during the first 10 decades of life: an autopsy study of 965 normal hearts. Mayo Clin Proc 1984; 59: 17-20.

4 Wilmshurst P, Pearson M, Nightingale S. Re-evaluation of the relationship between migraine and persistent foramen ovale and other right-to-left shunts. Clin Sci 2005; 108: 365-367.

5 Hirth A, Nightingale S, Wilmshurst P, Disney P, Thorne S. Prevalence of migraine in adults with cyanotic congenital heart disease. Cong Heart Dis 2008; 3: 124-127.

6 Steele JG, Nath PU, Burn J, Porteous MEM. An association between migrainous aura and hereditary haemorrhagic telangiectasia. Headache 1993; 33: 145-148.

7 Wilmshurst P, Nightingale S. The role of cardiac and pulmonary pathology in migraine: a hypothesis. Headache 2006; 46: 429-434.

8 Etminan M, Takkoucke B, Isorna FC, Samii A. Risk of ischaemic stroke in people with migraine: systematic review and meta-analysis of observational studies. BMJ 2005; 330: 63-65.

9 Wilmshurst P, Bryson P. Relationship between the clinical features of neurological decompression illness and its causes. Clin Sci 2000; 99: 65-75.

10 Wilmshurst PT, Pearson MJ, Walsh KP, Morrison WL. Relationship between right-to-left shunts and cutaneous decompression illness. Clin Sci 2001; 100: 539-542.

11 Engel GL, Webb JP, Ferris EB, Romano J, Ryder H, Blankenhorn MA. A migraine-like syndrome complicating decompression sickness. War Med 1944; 5: 304-314.

12 Smart D, Mitchell S, Wilmshurst P, Turner M, Banham N. Joint position statement on persistent foramen ovale and diving: South Pacific Underwater Medicine Society (SPUMS) and the United Kingdom Sports Diving Medical Committee (UKSDMC). Diving Hyperb Med 2015; 45: 129-131.

13 Wilmshurst PT, Morrison WL, Walsh KP, Pearson MJ, Nightingale S. Comparison of the size of persistent foramen ovale and atrial septal defects in divers with shunt-related decompression illness and in the general population. Diving Hyperb Med 2015; 45: 89-93.
14 Wilmshurst PT, Pearson MJ, Nightingale S, Walsh KP, Morrison WL. Inheritance of persistent foramen ovale and atrial septal defects and the relationship to familial migraine with aura. Heart 2004; 90: 1315-1320.

15 Wilmshurst P, Panikkar J, Pearson M. Nightingale S. Relation between inheritance of cyanotic congenital heart disease and persistent foramen ovale. Am J Cardiol 2009; 104: 148-149.

16 Wilmshurst PT, Nightingale S, Walsh KP, Morrison WL. Effect on migraine of closure of cardiac right-to-left shunts to prevent recurrence of decompression illness or stroke or for haemodynamic reasons. Lancet 2000; 356: 1648-1651.

17 Sztajzel R, Genoud D, Roth S, Mermillod B, Le Floch-Rohr J. Patent foramen ovale, a possible cause of symptomatic migraine: A study of 74 patients with acute ischemic stroke. Cerebrovasc Dis 2002; 13: 102-106.

18 Morandi E, Anzola GP, Angeli S, Melzi G, Onorato E. Transcatheter closure of patent foramen ovale: a new migraine treatment. J Interven Cardiol 2003; 16: 39-42.

19 Post MC, Thijs V, Herroelen L, Budts WIHL. Closure of a patent foramen ovale is associated with a decrease in prevalence of migraine. Neurology 2004; 62: 1439-1440.

20 Schwerzmann M, Wiher S, Nedeltchev K, Mattle HP, Wahl A, Seiler C et al. Percutaneous closure of patent foramen ovale reduces the frequency of migraine attacks. Neurology 2004; 62: 1399-1401.

21 Reisman M, Christofferson RD, Jesurum J, Olsen JV, Spencer MP, Krabill KA et al. Migraine headache relief after transcatheter closure of patent foramen ovale. J Am Coll Cardiol 2005; 45: 493-495.

22 Post MC, Thijs V, Schonenville WJ, Budts W, Snijder RJ et al. Embolization of pulmonary arteriovenous malformations and decrease in prevalence of migraine. Neurology 2006; 66: 202-205.

23 Bateman DN. Triptans and migraine. Lancet 2000; 355: 860-861.

24 Gaddum JH, Hebb CO, Silver A, Swan AAB. 5-Hydroxytryptamine; pharmacological action and destruction in perfused lungs. Q J Exp Physiol 1953; 38: 255-262.

25 Peto R, Gray R, Collins R, Wheatley K, Hennekens C, Jamrozik K et al. Randomised trial of prophylactic daily aspirin in British male doctors. BMJ 1988; 296: 313-316.

26 Wilmshurst PT, Nightingale S, Walsh KP, Morrison WL. Clopidogrel reduces migraine with aura following transcatheter closure of persistent foramen ovale and atrial septal defects. Heart 2005; 91: 1173-1175.

27 Dowson A, Mullen MJ, Peatfield R, Muir K, Khan AA, Wells $C$ et al. Migraine Intervention with STARFlex Technology (MIST) Trial. A prospective, multicenter, double-blind, sham-controlled trial to evaluate the effectiveness of patent foramen ovale closure with STARFlex septal repair implant to resolve refractory migraine headache. Circulation 2008; 117: 1397-1404.

28 Dyer C. Libel case against Wilmshurst collapses as firm goes into liquidation. BMJ 2011; 342: d2646.

29 Dyer C. Migraine doctor is suspended for serious breach of standards. BMJ 2015; 350: h982. 